\title{
On the $\boldsymbol{X}$-coordinates of Pell equations which are rep-digits, II
}

\author{
Florian Luca ${ }^{a}$, Sossa Victorin Togan ${ }^{b}$, Alain Togbé ${ }^{c}$
}

\author{
${ }^{a}$ School of Mathematics, University of the Witwatersrand, South Africa and Department \\ of Mathematics, Faculty of Sciences, University of Ostrava, Czech Republic \\ florian.luca@wits.ac.za \\ ${ }^{b}$ Institut de Mathématiques et de Sciences Physiques, Porto-Novo, Bénin \\ tofils740yahoo.fr \\ ${ }^{c}$ Department of Mathematics, Statistics and Computer Science \\ Purdue University Northwest,Westville,USA \\ atogbe@pnw.edu \\ Submitted: June 27, 2018 \\ Accepted: December 12, 2018 \\ Published online: February 11, 2019
}

\begin{abstract}
For a positive integer $d$ which is not a square, we show that there is at most one value of the positive integer $X$ participating in the Pell equation $X^{2}-d Y^{2}= \pm 4$ which is a rep-digit, that is all its base 10 digits are equal, except for $d=2,5,13$.
\end{abstract}

Keywords: Pell equation, Rep-digit, Linear forms in complex logarithms.

MSC: 11A25 11B39, 11J86

\section{Introduction}

Let $d$ be a positive integer which is not a perfect square. It is well-known that the Pell equation

$$
X^{2}-d Y^{2}= \pm 4
$$

has infinitely many positive integer solutions $(X, Y)$. Furthermore, putting $\left(X_{1}, Y_{1}\right)$ for the smallest such solution (solution with minimal value for $X$ ), all the positive 
integer solutions are of the form $\left(X_{n}, Y_{n}\right)$ for some positive integer $n$ where

$$
\frac{X_{n}+\sqrt{d} Y_{n}}{2}=\left(\frac{X_{1}+\sqrt{d} Y_{1}}{2}\right)^{n}
$$

There are many papers in the literature which solve Diophantine equations involving members of the sequences $\left\{X_{n}\right\}_{n \geq 1}$ or $\left\{Y_{n}\right\}_{n \geq 1}$ being squares, or perfect powers of larger exponents of some other integers, etc. (see, for example, $[4,5]$ ).

Let $g \geq 2$ be an integer. A natural number $N$ is called a base $g$ rep-digit if all of its base $g$-digits are equal; that is, if

$$
N=a\left(\frac{g^{m}-1}{g-1}\right), \quad \text { for some } \quad m \geq 1 \text { and } a \in\{1,2, \ldots, g-1\} .
$$

When $g=10$, we omit the base and simply say that $N$ is a rep-digit. Diophantine equations involving rep-digits were also considered in several papers which found all rep-digits which are perfect powers, or Fibonacci numbers, or generalized Fibonacci numbers, and so on (see $[1-3,7,9,11-15,17]$ for a sample of such results). In this paper, we study when can $X_{n}$ be a rep-digit. This reduces to the Diophantine equation

$$
X_{n}=a\left(\frac{10^{m}-1}{9}\right), \quad m \geq 1 \text { and } a \in\{1, \ldots, 9\} .
$$

Of course, for every positive integer $X$, there is a unique square-free integer $d \geq 2$ such that

$$
X^{2}-d Y^{2}=-4
$$

Namely $d$ is the product of all prime factors of $X^{2}+4$ which appear at odd exponents in its factorization. In particular, taking $X=a\left(10^{m}-1\right) / 9$, we get that any repdigit is the $X$-coordinate of the Pell equation (1.1) corresponding to some specific square-free integer $d$. If $X>2$, we can instead look at $X^{2}-4$ and write it as $d Y^{2}$ for some positive integers $d$ and $Y$ with $d$ squarefree, and then

$$
X^{2}-d Y^{2}=4 \text {. }
$$

In particular, we can take $X=a\left(10^{m}-1\right) / 9$ with $a \in\{1, \ldots, 9\}$ and $m \geq 1$, where we ask in addition that $a \geq 3$ when $m=1$. Here, we study the square-free integers $d$ such that the sequence $\left\{X_{n}\right\}_{n \geq 1}$ contains at least two rep-digits. Our result is the following.

Theorem 1.1. Let $d \geq 2$ be square-free. The Diophantine equation

$$
X_{n}=a\left(\frac{10^{m}-1}{9}\right), \quad m \geq 1 \text { and } a \in\{1, \ldots, 9\}
$$

has at most one positive integer solution $n$ except when $d=2,5,13$ for which we have

$$
2^{2}-2 \cdot 2^{2}=-4, \quad 6^{2}-2 \cdot 4^{2}=4,
$$

$1^{2}-5 \cdot 1^{2}=-4, \quad 3^{2}-5 \cdot 1^{2}=4, \quad 4^{2}-5 \cdot 2^{2}=-4 \quad 7^{2}-5 \cdot 3^{2}=4, \quad 11^{2}-5 \cdot 5^{2}=-4$, and

$$
3^{2}-13 \cdot 1^{2}=-4, \quad 11^{2}-13 \cdot 3^{2}=4
$$




\section{Linear forms in logarithms}

We need some results from the theory of lower bounds for nonzero linear forms in logarithms of algebraic numbers. We start by recalling Theorem 9.4 of [4], which is a modified version of a result of Matveev [16]. Let $\mathbb{L}$ be an algebraic number field of degree $d_{\mathbb{L}}$. Let $\eta_{1}, \eta_{2}, \ldots, \eta_{l} \in \mathbb{L}$ not 0 or 1 and $d_{1}, \ldots, d_{l}$ be nonzero integers. We put

$$
D=\max \left\{\left|d_{1}\right|, \ldots,\left|d_{l}\right|, 3\right\}
$$

and

$$
\Gamma=\prod_{i=1}^{l} \eta_{i}^{d_{i}}-1
$$

Let $A_{1}, \ldots, A_{l}$ be positive integers such that

$$
A_{j} \geq h^{\prime}\left(\eta_{j}\right):=\max \left\{d_{\mathbb{L}} h\left(\eta_{j}\right),\left|\log \eta_{j}\right|, 0.16\right\}, \quad \text { for } \quad j=1, \ldots l,
$$

where for an algebraic number $\eta$ of minimal polynomial

$$
f(X)=a_{0}\left(X-\eta^{(1)}\right) \cdots\left(X-\eta^{(k)}\right) \in \mathbb{Z}[X]
$$

over the integers with positive $a_{0}$, we write $h(\eta)$ for its Weil height given by

$$
h(\eta)=\frac{1}{k}\left(\log a_{0}+\sum_{j=1}^{k} \max \left\{0, \log \left|\eta^{(j)}\right|\right\}\right) .
$$

The following consequence of Matveev's theorem is Theorem 9.4 in [4].

Theorem 2.1. If $\Gamma \neq 0$ and $\mathbb{L} \subseteq \mathbb{R}$, then

$$
\log |\Gamma|>-1.4 \cdot 30^{l+3} l^{4.5} d_{\mathbb{L}}^{2}\left(1+\log d_{\mathbb{L}}\right)(1+\log D) A_{1} A_{2} \cdots A_{l} .
$$

When $l=2$ and $\eta_{1}, \eta_{2}$ are positive and multiplicatively independent, we can do better. Namely, let in this case $B_{1}, B_{2}$ be real numbers larger than 1 such that

$$
\log B_{i} \geq \max \left\{h\left(\eta_{i}\right), \frac{\left|\log \eta_{i}\right|}{d_{\mathbb{L}}}, \frac{1}{d_{\mathbb{L}}}\right\} \quad i=1,2,
$$

and put

$$
b^{\prime}:=\frac{\left|d_{1}\right|}{d_{\mathbb{L}} \log B_{2}}+\frac{\left|d_{2}\right|}{d_{\mathbb{L}} \log B_{1}} .
$$

Furthermore, let

$$
\Lambda=d_{1} \log \eta_{1}+d_{2} \log \eta_{2} .
$$

Note that $\Lambda \neq 0$ when $\eta_{1}$ and $\eta_{2}$ are multiplicatively independent. 
Theorem 2.2. With the above notations, assuming that $\mathbb{L}$ is real, $\eta_{1}, \eta_{2}$ are positive and multiplicatively independent, then

$$
\log |\Lambda|>-24.34 d_{\mathbb{L}}^{4}\left(\max \left\{\log b^{\prime}+0.14, \frac{21}{d_{\mathbb{L}}}, \frac{1}{2}\right\}\right)^{2} \log B_{1} \log B_{2} .
$$

Note that $e^{\Lambda}-1=\Gamma$, so $\Gamma$ is close to zero if and only if $\Lambda$ is close to zero, which explains the relation between Theorems 2.1 and 2.2 .

\section{The Baker-Davenport lemma}

Here, we recall the Baker-Davenport reduction method (see [8, Lemma 5a]), which turns out to be useful in order to reduce the bounds arising from applying Theorems 2.1 and 2.2 .

Lemma 3.1. Let $\kappa \neq 0$ and $\mu$ be real numbers. Assume that $M$ is a positive integer. Let $P / Q$ be the convergent of the continued fraction expansion of $\kappa$ such that $Q>6 M$ and put

$$
\xi=\|\mu Q\|-M \cdot\|\kappa Q\|,
$$

where $\|\cdot\|$ denotes the distance from the nearest integer. If $\xi>0$, then there is no solution to the inequality

$$
0<|m \kappa-n+\mu|<A B^{-k}
$$

in positive integers $m, n$ and $k$ with

$$
\frac{\log (A Q / \xi)}{\log B} \leq k \quad \text { and } \quad m \leq M
$$

\section{Bounding the variables}

We assume that $\left(X_{1}, Y_{1}\right)$ is the minimal solution of the Pell equation (1.1). Set

$$
X_{1}^{2}-d Y_{1}^{2}=: \pm 4
$$

and

$$
x_{n}=\frac{X_{n}}{2}, \quad y_{n}=\frac{Y_{n}}{2} \quad \text { for all } \quad n \geq 1
$$

We have

$$
x_{n}^{2}-d y_{n}^{2}=: \varepsilon_{n}, \quad \varepsilon_{n} \in\{ \pm 1\} .
$$

Put

$\delta:=x_{1}+\sqrt{x_{1}^{2}-\varepsilon_{1}}=x_{1}+\sqrt{d} y_{1}, \quad \eta:=x_{1}-\sqrt{d} y_{1}=\varepsilon_{1} \delta^{-1}, \quad$ with $\quad \delta \geq(1+\sqrt{5}) / 2$. 
Then, we get

$$
x_{n}=\frac{1}{2}\left(\delta^{n}+\eta^{n}\right),
$$

or, equivalently,

$$
X_{n}=\delta^{n}+\eta^{n} .
$$

We start with some general considerations concerning equation (1.2). From equation (1.2), we have

$$
X_{n}=a\left(\frac{10^{m}-1}{9}\right)>a\left(1+10+\cdots+10^{m-1}\right)>10^{m-1} .
$$

We get

$$
10^{m-1} \leq X_{n}<10^{m}
$$

Furthermore,

$$
2 \delta^{n}>\delta^{n}+\eta^{n}=X_{n} \geq \delta^{n}-\delta^{-n} \geq \frac{\delta^{n}}{2},
$$

where the last inequality follows because $n \geq 1$ and $\delta \geq(1+\sqrt{5}) / 2>\sqrt{2}$. So,

$$
\frac{\delta^{n}}{2} \leq X_{n}<2 \delta^{n} \quad \text { holds for all } n \geq 1
$$

Using now the equations (4.1) and (4.2), we have

$$
10^{m-1} \leq X_{n}<2 \delta^{n} \quad \text { and } \quad \frac{\delta^{n}}{2} \leq X_{n} \leq 10^{m}
$$

Hence, we obtain

$$
n c_{1} \log \delta-c_{2} \leq m \leq n c_{1} \log \delta+c_{2}+1, \quad c_{1}:=1 / \log 10, \quad c_{2}:=c_{1} \log 2 .
$$

From the left-hand side inequality of (4.3), we also deduce that

$$
n \log \delta<m \log 10+\log 2 .
$$

Since $\delta \geq(1+\sqrt{5}) / 2$, we get that

$$
n \leq m \frac{\log 10}{\log ((1+\sqrt{5}) / 2)}+\frac{\log 2}{\log ((1+\sqrt{5}) / 2)}<4.8 m+2 .
$$

If $m \geq 2$, the last inequality above implies that $n<6 m$. If $m=1$, then $X_{n} \leq 9$, so $\delta^{n} \leq 18$ by (4.2). Since $\delta \geq(1+\sqrt{5}) / 2$, we get that $n \leq 6$, so the inequality $n \leq 6 m$ holds also when $m=1$. We record this as

$$
n \leq 6 m \text {. }
$$

Next, using (1.3), we get

$$
\delta^{n}+\eta^{n}=a\left(\frac{10^{m}-1}{9}\right)
$$


Put $b:=a / 9$. We have

$$
\delta^{n} b^{-1} 10^{-m}-1=-b^{-1} 10^{-m} \eta^{n}-10^{-m} .
$$

Thus,

$$
\begin{aligned}
\left|\delta^{n} b^{-1} 10^{-m}-1\right| & \leq \frac{1}{b 10^{m} \delta^{n}}+\frac{1}{10^{m}}=\frac{1}{10^{m}}\left(1+\frac{9}{a \delta^{n}}\right) \\
& <\frac{6}{10^{m}}
\end{aligned}
$$

using that $a \geq 1, n \geq 1$ and $\delta \geq(1+\sqrt{5}) / 2$. Thus,

$$
\left|\delta^{n} b^{-1} 10^{-m}-1\right|<\frac{6}{10^{m}}
$$

We now assume that $m \geq 2$ and search for an upper bound on it. Since $m \geq 2$, it follows that the right-hand side in (4.6) above is $<1 / 2$. Put

$$
\Lambda:=n \log \delta-\log b-m \log 10 .
$$

Since $\left|e^{\Lambda}-1\right|<1 / 2$, it follows that

$$
|\Lambda|<2\left|e^{\Lambda}-1\right|<\frac{12}{10^{m}}
$$

Let us return to (4.6) and put

$$
\Gamma:=e^{\Lambda}-1=\delta^{n} b^{-1} 10^{-m}-1 .
$$

Note that $\Gamma$ is nonzero. Indeed, if it were zero, then $\delta^{n}=b 10^{m}$. Hence, $\delta^{n} \in \mathbb{Q}$. Since $\delta$ is an algebraic integer and $n \geq 1$, it follows that $\delta^{n} \in \mathbb{Z}$. Since $\delta$ is a unit, we get that $\delta^{n}=1$, so $n=0$, which is a contradiction. Thus, $\Gamma \neq 0$. We apply Matveev's theorem. If $a \neq 9$ (so, $b \neq 1)$, we then take

$$
l=3, \eta_{1}=\delta, \eta_{2}=b, \eta_{3}=10, d_{1}=n, d_{2}=-1, d_{3}=-m, D=\max \{n, m\} .
$$

Clearly, $\mathbb{L}=\mathbb{Q}[\sqrt{d}]$ contains all the numbers $\eta_{1}, \eta_{2}, \eta_{3}$ and has degree $d_{\mathbb{L}}=2$. We have

$$
h\left(\eta_{1}\right)=(1 / 2) \log \delta, \quad h\left(\eta_{2}\right) \leq \log 9 \quad \text { and } \quad h\left(\eta_{3}\right)=\log 10 .
$$

Thus, we can take

$$
A_{1}=\log \delta, \quad A_{2}=2 \log 9 \quad \text { and } \quad A_{3}=2 \log 10 .
$$

Now, Theorem 2.1 tells us that

$$
\log |\Gamma|>-1.4 \times 30^{6} \times 3^{4.5} \times 2^{2}(1+\log 2)(1+\log D)(\log \delta)(2 \log 9)(2 \log 10) .
$$


Comparing the above inequality with (4.6), we get $m \log 10-\log 6<1.4 \times 30^{6} \times 3^{4.5} \times 2^{4}(1+\log 2)(1+\log D)(\log \delta)(\log 9)(\log 10)$. Thus,

$$
m<1.4 \times 30^{6} \times 3^{4.5} \times 2^{4} \times(\log 9)(1+\log 2) \times(\log \delta) \cdot(1+\log D)
$$

or

$$
m<8.6 \cdot 10^{12}(1+\log D) \log \delta .
$$

Since $D \leq 6 m$ (see $(4.5))$, we get

$$
m<8.6 \cdot 10^{12}(1+\log (6 m)) \log \delta .
$$

This was when $b \neq 1$. In case $b=1$, we take $l=2$ and apply the same inequality (except that now $\eta_{2}:=1$ is no longer present) getting a better result. Finally, this was under the assumption that $m \geq 2$ but if $m=1$ then inequality (4.7) also holds. Let us record what we have proved so far.

Lemma 4.1. Denoting by $\delta:=x_{1}+\sqrt{d} y_{1}$, all positive integer solutions $(m, n)$ of equation (1.2) satisfy

$$
m<8.6 \cdot 10^{12}(1+\log (6 m)) \log \delta .
$$

All this is for the equation $X_{n}=a\left(10^{m}-1\right) / 9$. Now we assume that

$$
X_{n_{1}}=a_{1}\left(\frac{10^{m_{1}}-1}{9}\right) \text { and } X_{n_{2}}=a_{2}\left(\frac{10^{m_{2}}-1}{9}\right) .
$$

where $a_{1}, a_{2} \in\{1, \ldots, 9\}$.

To fix ideas, we assume that $n_{1}<n_{2}$, so $m_{1} \leq m_{2}$. We put as before $b_{i}:=a_{i} / 9$ for $i=1,2$. From the above analysis, assuming that $m_{1} \geq 2$, we have that

$$
\left|n_{i} \log \delta-\log b_{i}-m_{i} \log 10\right|<\frac{12}{10^{m_{i}}} \quad \text { holds for } i \in\{1,2\} .
$$

The argument proceeds in two steps according to whether $b_{1} b_{2}<1$ or $b_{1} b_{2}=1$.

Suppose now that $b_{1} b_{2}<1$.

We multiply the equation (4.8) for $i=1$ with $n_{2}$ and the one for $i=2$ with $n_{1}$, subtract them and apply the absolute value inequality to get

$$
\begin{aligned}
& \left|n_{2} \log b_{1}-n_{1} \log b_{2}+\left(n_{2} m_{1}-n_{1} m_{2}\right) \log 10\right| \\
& =\left|n_{1}\left(n_{2} \log \delta-\log b_{2}-m_{2} \log 10\right)-n_{2}\left(n_{1} \log \delta-\log b_{1}-m_{1} \log 10\right)\right| \\
& \leq n_{1}\left|n_{2} \log \delta-\log b_{1}-m_{2} \log 10\right|+n_{2}\left|n_{1} \log \delta-\log b_{1}-m_{1} \log 10\right| \\
& \leq \frac{12 n_{1}}{10^{m_{2}}}+\frac{12 n_{2}}{10^{m_{1}}} \leq \frac{24 n_{2}}{10^{m_{1}}} .
\end{aligned}
$$


If the right-hand side above is at least $1 / 2$, we then get

$$
10^{m_{1}} \leq 48 n_{2}<300 m_{2}
$$

giving

$$
m_{1}<c_{1} \log \left(300 m_{2}\right)
$$

Assume now that the right-hand side in (4.9) is smaller than $1 / 2$. Putting,

$$
\Lambda_{0}:=n_{2} \log b_{1}-n_{1} \log b_{2}+\left(n_{2} m_{1}-n_{1} m_{2}\right) \log 10,
$$

we get $\left|\Lambda_{0}\right|<1 / 2$. Putting

$$
\Gamma_{0}:=b_{1}^{n_{2}} b_{2}^{-n_{1}} 10^{n_{2} m_{1}-n_{1} m_{2}}-1,
$$

we get that

$$
\left|\Gamma_{0}\right|=\left|e^{\Lambda_{0}}-1\right|<2\left|\Lambda_{0}\right|<\frac{48 n_{2}}{10^{m_{1}}},
$$

where the middle inequality above follows from the fact that $\left|\Lambda_{0}\right|<1 / 2$. We apply Matveev's theorem to estimate a lower bound on $\Gamma_{0}$. But first, let us see that it is nonzero. Assuming $\Gamma_{0}=0$, we get

$$
b_{1}^{n_{2}} b_{2}^{-n_{1}}=10^{n_{2} m_{1}-n_{1} m_{2}} .
$$

Assume first that $n_{2} m_{1}-n_{1} m_{2}=0$. Then $b_{1}^{n_{2}}=b_{2}^{n_{1}}$. Thus, $b_{1}$ and $b_{2}$ are multiplicatively independent and they belong to the set

$$
\left\{\frac{1}{9}, \frac{2}{9}, \frac{1}{3}, \frac{4}{9}, \frac{5}{9}, \frac{2}{3}, \frac{7}{9}, \frac{8}{9}, 1\right\} .
$$

They are not both 1 and $n_{1}$ and $n_{2}$ are both positive. So, the only possibilities are that $b_{1}=b_{2}$, or

$$
\left\{b_{1}, b_{2}\right\}=\left\{\frac{1}{9}, \frac{1}{3}\right\},\left\{\frac{2}{3}, \frac{4}{9}\right\} .
$$

If $b_{1}=b_{2}$, then $b_{1}^{n_{1}}=b_{2}^{n_{2}}$ implies $n_{1}=n_{2}$, which together with $n_{2} m_{1}=n_{1} m_{2}$ leads to $m_{1}=m_{2}$. Thus, $\left(n_{1}, m_{1}\right)=\left(n_{2}, m_{2}\right)$ and $a_{1}=a_{2}$ (because $\left.b_{1}=b_{2}\right)$, and this is not convenient for us. If $\left\{b_{1}, b_{2}\right\}$ is one of the two sets from (4.13), then one of $b_{1}, b_{2}$ is the square of the other one. Thus, since $b_{1}^{n_{1}}=b_{2}^{n_{2}}$ and $n_{2}>n_{1}$, we get $n_{2}=2 n_{1}$. Since also $n_{2} m_{1}=n_{1} m_{2}$, we have $m_{2}=2 m_{1}$. Hence, also $b_{2}=b_{1}^{2}$ and $b_{1} \in\{1 / 3,2 / 3\}$. So, we get the pair of equations

$$
X_{n_{1}}=b_{1} 10^{m_{1}}-b_{1} \quad \text { and } \quad X_{2 n_{1}}=b_{1}^{2} 10^{2 m_{1}}-b_{1}^{2} .
$$

Since in fact

$$
X_{2 n}=\delta^{2 n}+\eta^{2 n}=\left(\delta^{n}+\eta^{n}\right)^{2}-2(\delta \eta)^{n}=X_{n}^{2} \pm 2
$$


we get that

$b_{1}^{2} 10^{2 m_{1}}-b_{1}^{2}=X_{2 n_{1}}=X_{n_{1}}^{2} \pm 2=\left(b_{1} 10^{m_{1}}-b_{1}\right)^{2} \pm 2=b_{1}^{2} 10^{2 m_{1}}-2 b_{1}^{2} 10^{m_{1}}+b_{1}^{2} \pm 2$,

which leads to

$$
2 b_{1}^{2} 10^{m_{1}}=2 b_{1}^{2} \pm 2,
$$

so

$$
10^{m_{1}}=1 \pm b_{1}^{-2}
$$

The last equation above is impossible for $m_{1} \geq 2$. For $m_{1}=1$ we get $10=1 \pm b_{1}^{-2}$, which gives $b_{1}=1 / 3$. Hence,

$$
X_{n_{1}}=\frac{10-1}{3}=3, \quad \text { and } \quad X_{2 n_{1}}=\frac{10^{2}-1}{9}=11 .
$$

Since $X_{2 n_{1}}=X_{n_{1}}^{2} \pm 2$, it follows that the sign is + , so $X_{n_{1}}^{2}-d Y_{n_{1}}^{2}=-4$, giving $d Y_{n_{1}}^{2}=13$, so $d=13, Y_{1}=1, n_{1}=1$. These solutions are among the ones mentioned in the statement of the main theorem.

This deals with the case when $n_{2} m_{1}-n_{1} m_{2}=0$. Assume next that $n_{2} m_{1}-$ $n_{1} m_{2} \neq 0$. Then in the right-hand side of (4.12), both primes 2 and 5 are involved at a nonzero exponent. Thus, they should be also involved with nonzero exponents in the left-hand side of (4.12). Thus, one of $b_{1}, b_{2}$ is $5 / 9$ and the other is in $\{2 / 9,4 / 9,2 / 3,8 / 9\}$. A minute of reflection shows that in all cases the exponents of 2 and 5 in the left-hand side of (4.12) have opposite signs, whereas in the right they have the same sign, and this is impossible.

Thus, $\Gamma_{0} \neq 0$. Hence, we are entitled to apply Matveev's theorem in order to find a lower bound on $\Gamma_{0}$. In case $b_{1} \neq 1$ and $b_{2} \neq 1$, we take

$$
l=3, \eta_{1}=b_{1}, \eta_{2}=b_{2}, \eta_{3}=10, d_{1}=n_{2}, d_{2}=-n_{1}, d_{3}=n_{2} m_{1}-n_{1} m_{2} .
$$

Clearly, $\mathbb{L}=\mathbb{Q}$ contains all the numbers $\eta_{1}, \eta_{2}, \eta_{3}$ and has degree $d_{\mathbb{L}}=1$. Further, $D=\max \left\{\left|d_{1}\right|,\left|d_{2}\right|,\left|d_{3}\right|\right\} \leq n_{2} m_{2} \leq 6 m_{2}^{2}$. We have

$$
h\left(\eta_{1}\right) \leq \log 9, \quad h\left(\eta_{2}\right) \leq \log 9 \quad \text { and } \quad h\left(\eta_{3}\right)=\log 10 .
$$

Thus, we can take

$$
A_{1}=\log 9, \quad A_{2}=\log 9, \quad A_{3}=\log 10 .
$$

Now, Theorem 2.1 tells us that

$$
\log \left|\Gamma_{0}\right|>-1.4 \times 30^{6} \times 3^{4.5}(1+\log D)(\log 9)^{2}(\log 10) .
$$

Combining this with estimate (4.11) and using the fact that $48 n_{2}<300 m_{2}$ (see inequality (4.5)) we get

$$
m_{1} \log 10 \leq \log 300+\log m_{2}+1.6 \times 10^{12}\left(1+\log \left(6 m_{2}^{2}\right)\right),
$$


giving

$$
m_{1}<7 \times 10^{11}\left(1+\log \left(6 m_{2}^{2}\right)\right) .
$$

The right-hand side of inequality (4.14) is larger than the right-hand side of inequality (4.10). So, regardless whether $24 n_{2} / 10^{m_{1}}$ is at least $1 / 2$ or smaller than $1 / 2$, estimate (4.14) holds. From equation (4.4), we get

$$
\log \delta<\left(m_{1}+1\right) \log 10<1.7 \times 10^{12}\left(1+\log \left(6 m_{2}^{2}\right)\right),
$$

which together with Lemma 4.1 gives

$$
m_{2}<\left(8.6 \times 10^{12}\left(1+\log \left(6 m_{2}\right)\right)\right)\left(1.7 \times 10^{12}\left(1+\log \left(6 m_{2}^{2}\right)\right)\right),
$$

So

$$
m_{2}<1.5 \times 10^{25}\left(1+\log \left(6 m_{2}\right)\right)\left(1+\log \left(6 m_{2}^{2}\right)\right) .
$$

This gives $m_{2}<1.5 \times 10^{29}$. This was if both $b_{1}$ and $b_{2}$ are different than 1 . If one of them is 1 , we simply apply Matveev's theorem with $l=2$ getting an even better bound for $m_{2}$.

Suppose now that $b_{1}=b_{2}=1$.

We return to (4.11) getting that $8 / 9 \leq 24 n_{2} / 10^{m_{1}}$, which leads to (4.10), unless $n_{1} m_{2}=n_{2} m_{1}$. In this last case, we get that $n_{2} / m_{2}=n_{1} / m_{1}$. Thus, writing $n_{1} / m_{1}=r / s$ in reduced terms, we get that $\left(n_{1}, m_{1}\right)=\left(\ell_{1} r, \ell_{1} s\right)$ and that $\left(n_{2}, m_{2}\right)=\left(\ell_{2} r, \ell_{2} s\right)$ for some positive integers $\ell_{1}<\ell_{2}$. Hence, we have

$$
X_{r \ell_{1}}=10^{s \ell_{1}}-1, \quad X_{r \ell_{2}}=10^{s \ell_{2}}-1 .
$$

The greatest common divisor of the right hand sides above is $10^{s}-1 \geq 9$. The greatest common divisor of the left-hand sides above is $X_{r}$ if $\ell_{1} \ell_{2}$ is odd and 1 or 2 otherwise. Thus, $\ell_{1} \ell_{2}$ must be odd and

$$
X_{r}=10^{s}-1
$$

Consequently,

$$
\delta^{r}-10^{s}=-\eta^{r}-1 \quad \text { and } \quad \delta^{\ell_{2} r}-10^{\ell_{2} s}=-\eta^{\ell_{2} r}-1 .
$$

From the two equations above we get

$$
\delta^{\left(\ell_{2}-1\right) r}+\delta^{\left(\ell_{2}-2\right)} 10^{s}+\cdots+10^{\left(\ell_{2}-1\right) s}=\frac{-\eta^{\ell_{2} r}-1}{-\eta^{r}-1} .
$$

The last relation above is impossible since its left-hand side is $>10$ and its right hand side is

$$
\leq \frac{2}{1-\frac{2}{1+\sqrt{5}}}<10
$$

a contradiction.

In conclusion, (4.10) holds, which is stronger than (4.14), and the above arguments imply that $m_{2}<1.5 \times 10^{29}$. Hence, we have the following result. 
Lemma 4.2. The inequality

$$
m_{2}<1.5 \times 10^{29}
$$

holds.

Now one needs to apply LLL to the bound

$$
\left|\Lambda_{0}\right|<\frac{24 n_{2}}{10^{m_{1}}}<\frac{24 \times 6 \times 1.5 \times 10^{29}}{10^{m_{1}}}<\frac{1}{10^{m_{1}-32}}
$$

to get a reasonably small bound on $m_{1}$.

- First, we will consider the case $b_{1}=b_{2}:=b$; i.e., $a_{1}=a_{2}:=a$ or

$$
\left\{b_{1}, b_{2}\right\} \in\left\{\frac{1}{9}, \frac{1}{3}\right\},\left\{\frac{2}{3}, \frac{4}{9}\right\} .
$$

In

$$
\Lambda_{0}:=n_{2} \log b_{1}-n_{1} \log b_{2}+\left(n_{2} m_{1}-n_{1} m_{2}\right) \log 10,
$$

we set $X:=n_{1}-n_{2}$ or $X:=2 n_{2}-n_{1}$, and $Y:=n_{2} m_{1}-n_{1} m_{2}$ and divide both sides by $Y \log b$ (with $b=b_{1}=b_{2} \in\{1 / 9,2 / 9,3 / 9,4 / 9,5 / 9,6 / 9,7 / 9,8 / 9\}$ ) to get

$$
\left|\frac{\log 10}{\log b}-\frac{X}{Y}\right|<\frac{1}{Y(\log (1 / b)) 10^{m_{1}-32}} .
$$

We assume that $m_{1}$ is so large that the right-hand side in (4.16) is smaller than $1 /\left(2 Y^{2}\right)$. This certainly holds if

$$
10^{m_{1}-32}>2(\log (1 / b))^{-1} Y .
$$

Since $|Y|<1.5 \times 10^{59}$, it follows that the last inequality (4.17) holds provided that $m_{1} \geq 92$ in all cases, which we now assume. In this case, $X / Y$ is a convergent of the continued fraction of $\eta:=\log 10 / \log b$ and $X<1.5 \times 10^{59}$. Writing

$$
\begin{aligned}
a & =1, \quad \eta:=[-2,1,19,1,5,1,6,2,5,15,3, \ldots, 7,2,121,1, \ldots, 2,569,1,2,27,7, \ldots] \\
a & =2, \quad \eta:=[-2,2,7,1,1,2,4,2,99, \ldots] 1,292,1,6,1,3,3,2,2,5, \ldots, 1,1,1,42, \ldots] \\
a & =3, \quad \eta:=[-3,1,9,2,2,1,13,1,7,18, \ldots, 2,10,3,1,1,1,1,1,6, \ldots, 1,284,2, \ldots] \\
a & =4, \quad \eta:=[-3,6,4,2,1,1,1,1,45,89,1,6,1,9,1,2,625, \ldots, 2,2,1,1716,1,1, \ldots] \\
a & =5, \quad \eta:=[-4,12,9,1,1,1,1,1,2,1, \ldots, 10,1,1,12,8860,4,13,1,1,5,3,9,1, \ldots] \\
a & =6, \quad \eta:=[-6,3,8,1,3,3,22,1,1,44, \ldots, 1,1,38,1,5,1,857,1,3,1,3,1,2,1, \ldots] \\
a & =7, \quad \eta:=[-10,1,5,6,118,2,8,1,2,1, \ldots, 8,23,1,30,2,2,8,1,4,2,1,1,255, \ldots] \\
a & =8, \quad \eta:=[-20,2,4,1,1,3,2,7,1,2,1,9,2,6, \ldots, 1,2,1332,1,12,1,5,1,1,2, \ldots]
\end{aligned}
$$


for the continued fraction of $\eta$ and $p_{k} / q_{k}$ for the $k$ th convergent, we get that $X / Y=p_{j} / q_{j}$ for some $j \leq 122$ in all cases. Furthermore, putting $M:=\max \left\{a_{j}\right.$ : $0 \leq j \leq 122\}$, we get $M=8860$ (for $a=5$ ). From the known properties of the continued fractions, we then get that

$$
\frac{1}{8862 Y^{2}}=\frac{1}{(M+2) Y^{2}} \leq\left|\eta-\frac{X}{Y}\right|<\frac{1}{Y(\log b) 10^{m_{1}-32}},
$$

giving

$$
10^{m_{1}-32}<8862(\log b)^{-1} Y<8862(\log b)^{-1}\left(1.5 \times 10^{59}\right),
$$

leading to $m_{1} \leq 96$.

- We now consider the remaining cases. We transform the linear form (4.15) into one of the following forms:

$$
\begin{gathered}
\Lambda_{1}=\begin{array}{c}
\left(m_{1} n_{2}-m_{2} n_{1}+\delta_{1} n_{1}+\delta_{2} n_{2}\right) \log 2+\left(\lambda_{1} n_{1}+\lambda_{2} n_{2}\right) \log 3 \\
+\left(m_{1} n_{2}-m_{2} n_{1}+\mu_{1} n_{1}+\mu_{2} n_{2}\right) \log 5
\end{array} \\
\Lambda_{2}:=\left(\lambda_{1} n_{1}+\lambda_{2} n_{2}\right) \log 3+\left(\nu_{1} n_{1}+\nu_{2} n_{2}\right) \log 7+\left(m_{1} n_{2}-m_{2} n_{1}\right) \log 10 \\
\Lambda_{3}=\quad \begin{array}{c}
\left(m_{1} n_{2}-m_{2} n_{1}+\delta_{1} n_{1}+\delta_{2} n_{2}\right) \log 2+\left(\lambda_{1} n_{1}+\lambda_{2} n_{2}\right) \log 3 \\
+ \\
+\left(m_{1} n_{2}-m_{2} n_{1}+\mu_{1} n_{1}+\mu_{2} n_{2}\right) \log 5+\left(\nu_{1} n_{1}+\nu_{2} n_{2}\right) \log 7
\end{array}
\end{gathered}
$$

where $\left|\delta_{i}\right| \leq 3,\left|\lambda_{i}\right| \leq 2,\left|\mu_{i}\right| \leq 1,\left|\nu_{i}\right| \leq 1$, for $i=1,2$.

Now, we will estimate lower bounds for $\Lambda_{i}, i=1,2,3$ via the LLL algorithm (see Proposition 2.3.20 in [6]). One knows that $\Lambda_{i} \neq 0, i=1,2,3$ by what is done above. We set $X_{1}=X_{3}:=10^{60}$ as upper bounds for $\left|m_{1} n_{2}-m_{2} n_{1}+\delta_{1} n_{1}+\delta_{2} n_{2}\right|$, $\left|m_{1} n_{2}-m_{2} n_{1}+\mu_{1} n_{1}+\mu_{2} n_{2}\right|$ and $X_{2}=X_{4}:=10^{31}$ as upper bounds for $\mid \lambda_{1} n_{1}+$ $\lambda_{2} n_{2}|,| \nu_{1} n_{1}+\nu_{2} n_{2} \mid$. We take $C:=\left(3 X_{1}\right)^{3}$ for $\Lambda_{1}, \Lambda_{2}$ and $C:=\left(4 X_{1}\right)^{4}$ for $\Lambda_{3}$. Moreover, we consider the lattice $\Omega$ spanned by

$$
v_{1}:=(1,0,\lfloor C \log 2\rfloor), \quad v_{2}:=(0,1,\lfloor C \log 3\rfloor), \quad v_{3}:=(0,0,\lfloor C \log 5\rfloor),
$$

for $\Lambda_{1}$

$$
v_{1}:=(1,0,\lfloor C \log 3\rfloor), \quad v_{2}:=(0,1,\lfloor C \log 7\rfloor), \quad v_{3}:=(0,0,\lfloor C \log 10\rfloor),
$$

for $\Lambda_{2}$

$$
\begin{array}{ll}
v_{1}:=(1,0,0,\lfloor C \log 2\rfloor), & v_{2}:=(0,1,0,\lfloor C \log 3\rfloor), \\
v_{3}:=(0,0,1,\lfloor C \log 5\rfloor), & v_{4}:=(0,0,0,\lfloor C \log 7\rfloor),
\end{array}
$$

for $\Lambda_{3}$. Then, we compute $Q, T, c_{1}, m$ according to Proposition 2.3.20 in [6] and we obtain:

$$
\begin{array}{lll}
5.5 \cdot 10^{-122}<\left|\Lambda_{1}\right|<\frac{1}{10^{m_{1}-32}} & \Rightarrow & m_{1} \leq 153 ; \\
3.2 \cdot 10^{-122}<\left|\Lambda_{2}\right|<\frac{1}{10^{m_{1}-32}} \quad \Rightarrow & m_{1} \leq 153 ;
\end{array}
$$




$$
8.1 \cdot 10^{-183}<\left|\Lambda_{3}\right|<\frac{1}{10^{m_{1}-32}} \quad \Rightarrow \quad m_{1} \leq 214 .
$$

Hence, we have the following numerical result.

Lemma 4.3. The estimate $m_{1} \leq 214$ holds.

For $a_{1} \in\{1,2, \ldots, 9\}, 1 \leq n_{1} \leq 1284,1 \leq m_{1} \leq 214$, we solve the equations

$$
x_{n_{1}}=P_{n_{1}}\left(x_{1}\right)=a_{1}\left(\frac{10^{m_{1}}-1}{9}\right)
$$

to see for which values of the triple $\left(n_{1}, m_{1}\right)$ it has a solution $x_{1}=X_{1} / 2$ with positive integer $X_{1}$, where

$$
x_{n}=P_{n}(X / 2)=\left(\frac{X+\sqrt{X^{2} \pm 4}}{2}\right)^{n}+\left(\frac{X-\sqrt{X^{2} \pm 4}}{2}\right)^{n} .
$$

We used a program written in Maple to see that $n_{1}=1$ in all cases. Here, $P_{n}(X)$ is one of the two polynomials giving $x_{n}$ in terms of $x_{1}$ for the equation $x^{2}-d y^{2}= \pm 4$.

From equation (4.8), for $i=2$ we get

$$
\left|n_{2} \frac{\log \delta}{\log 10}-\frac{\log b_{2}}{\log 10}-m_{2}\right|<\frac{12}{(\log 10) 10^{m_{2}}},
$$

where $\delta=x_{1}+y_{1} \sqrt{d}=x_{1}+\sqrt{x_{1}^{2} \pm 4}, x_{1}=a_{1}\left(10^{m_{1}}-1\right) / 9$, and $b_{2}=a_{2} / 9$ with $a_{1} \neq a_{2}$. To apply Lemma 3.1 to inequality (4.18), we put

$$
\kappa=\frac{\log \delta}{\log 10}, \quad \mu=\frac{\log b_{2}}{\log 10}, \quad A=\frac{12}{\log 10}, \quad B=10, \quad \text { and } \quad M=1.5 \cdot 10^{29} .
$$

The program was developed in PARI/GP running with 200 digits, for $1 \leq m_{1} \leq$ 214. For the computations, if the first convergent such that $q>6 M$ does not satisfy the condition $\eta>0$, then we use the next convergent until we find the one that satisfies the conditions. In a few minutes, all the computations were done. In all cases, after the first run we obtained $m_{2} \leq 35$. We set $M=35$ and the second run of the reduction method yields $m_{2} \leq 8$. In conclusion, we have

$$
n_{1}=1, \quad 1 \leq m_{1} \leq 8, \quad 1 \leq m_{2} \leq 8, \quad 1 \leq n_{2} \leq 48
$$

Now a verification by hand yields the final result.

Acknowledgements. F. L. was supported in part by grant CPRR160325161141 and an A-rated scientist award both from the NRF of South Africa and by grant no. 17-02804S of the Czech Granting Agency. This paper was finalized during a visit of A.T. at the School of Mathematics of Wits University in August 2017. This author thanks this institution for its hospitality and the CoEMaSS at Wits for support. 


\section{References}

[1] S. D. Alvarado, F. Luca: Fibonacci numbers which are sums of two repdigits, in: Proceedings of the XIVth International Conference on Fibonacci numbers and their applications, vol. 20, Sociedad Matematica Mexicana, Aportaciones Matemáticas, Investigación, 2011, pp. 97-108.

[2] J. J. Bravo, F. Luca: On a conjecture about repdigits in k-generalized Fibonacci sequences, Publ. Math. Debrecen 82 (2013), pp. 623-639, DOI: 10.5486/pmd.2013.5390.

[3] Y. Bugeaud, M. Mignotte: On integers with identical digits, Mathematika 46 (1999), pp. 411-417, DOI: $10.1112 / \mathrm{s} 0025579300007865$.

[4] Y. Bugeaud, M. Mignotte, S. Siksek: Classical and modular approaches to exponential Diophantine equations I. Fibonacci and Lucas perfect powers, Annals of Mathematics 163 (2006), pp. 969-1018, DOI: 10.4007/annals.2006.163.969.

[5] Y. Bugeaud, P. Minăilescu: On the Nagell-Ljunggren equation $\left(x^{n}-1\right) /(x-1)=y^{q}$, Math. Scand. 101 (2007), pp. 177-183, DoI: 10.7146/math.scand.a-15038.

[6] H. Cohen: Number Theory, Vol. I: Tools and Diophantine Equations, New York: Springer, 2007, DOI: $10.5860 /$ choice.45-2655.

[7] A. Dossavi-Yovo, F. Luca, A. Togbé: On the $x$-coordinates of Pell equations which are rep-digits, Publ. Math. Debrecen 88 (2016), pp. 381-391, DOI: 10.5486/pmd.2016.7378.

[8] A. Dujella, A. Pethö: A generalization of a theorem of Baker and Davenport, Quart. J. Math. 49.195 (1998), pp. 291-306, DOI: 10.1093/qjmath/49.195.291.

[9] B. FAYE, F. LuCA: On x-coordinates of Pell equations which are repdigits, Fibonacci Quart. 56 (2018), pp. 52-62.

[10] M. Laurent, M. Mignotte, Y. Nesterenko: Formes linéaires en deux logarithmes et déterminants d'interpolation, J. Number Theory 55 (1995), pp. 285-321, DoI: 10.1006 / jnth.1995.1141.

[11] F. LucA: Fibonacci and Lucas numbers with only one distinct digit, Port. Math. 57 (2000), pp. 243-254.

[12] F. LucA: Repdigits which are sums of at most three Fibonacci number, Math. Comm. 17 (2012), pp. 1-11.

[13] F. Luca, A. Togbé: On the $x$-coordinates of Pell equations which are Fibonacci numbers, Math. Scand. 122 (2018), pp. 18-30, DOI: 10.7146/math.scand.a-97271.

[14] D. Marques, A. Togbé: On repdigits as product of consecutive Fibonacci numbers, Rend. Istit. Mat. Univ. Trieste 44 (2012), pp. 393-397.

[15] D. Marques, A. TogBÉ: On terms of linear recurrence sequences with only one distinct block of digits, Colloq. Math. 124 (2011), pp. 145-155, DOI: 10.4064/cm124-2-1.

[16] E. M. Matveev: An explicit lower bound for a homogeneous rational linear form in logarithms of algebraic numbers, II, Izv. Math. 64 (2000), pp. 1217-1269, DOI: 10.1070 / im2000v064n06abeh000314.

[17] R. ОвцÁтн: Une propriété des puissances parfaites, Mathesis 65 (1956), pp. 356-364.

[18] W. R. Spickerman: Binet's formula for the Tribonacci numbers, Fibonacci Quart. 20 (1982), pp. 118-120.

[19] K. YU: p-adic logarithmic forms and group varieties II, Acta Arith. 89 (1999), pp. 337-378, DOI: $10.4064 / \mathrm{aa}-89-4-337-378$. 